Braz J Med Biol Res, December 2011, Volume 44(12) 1222-1230

doi: 10.1590/S0100-879X2011007500143

Inhibition of STAT3 by RNA interference suppresses angiogenesis in colorectal carcinoma

W.F. Qian, W.X. Guan, Y. Gao, J.F. Tan, Z.M. Qiao, H. Huang and C.L. Xia

The Brazilian Journal of Medical and Biological Research is partially financed by

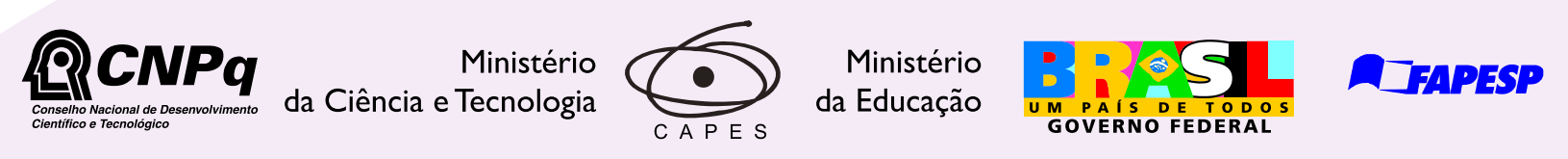

Institutional Sponsors
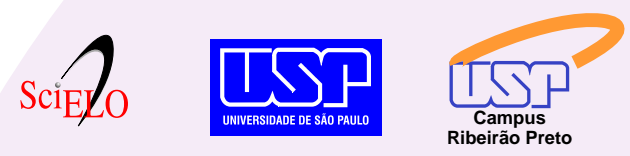

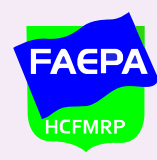

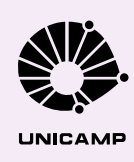

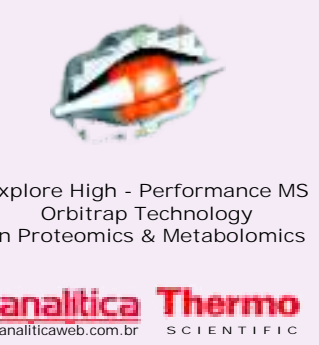




\title{
Inhibition of STAT3 by RNA interference suppresses angiogenesis in colorectal carcinoma
}

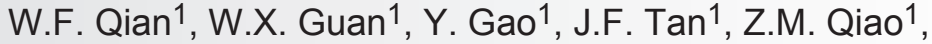 \\ H. Huang ${ }^{1}$ and C.L. Xia ${ }^{2}$ \\ ${ }^{1}$ Department of General Surgery, Suzhou Hospital Affiliated to Nanjing Medical University, \\ Suzhou, JS, China \\ ${ }^{2}$ Department of Anatomy and Cytoneurobiology Laboratory, Medical College, Soochow University, \\ Suzhou Industrial Park, Suzhou, JS, China
}

\begin{abstract}
In order to investigate signal transduction and activation of transcription 3 (STAT3) signaling on angiogenesis in colorectal carcinoma (CRC) after inhibiting STAT3 expression, we constructed the HT-29-shSTAT3 cell line by lentivirus-mediated RNAi. Cell growth was assessed with MTT and the cell cycle distribution by flow cytometry. CRC nude mouse models were established and tumor growth was monitored periodically. On day 30, all mice were killed and tumor tissues were removed. Microvessel density (MVD) was determined according to CD34-positive staining. The expression of vascular endothelial growth factor A (VEGFA), matrix metalloproteinase-2 (MMP2) and basic fibroblast growth factor (FGF2) was monitored by quantitative real-time PCR and Western blot analysis. Knockdown of STAT3 expression significantly inhibited cell growth in HT-29 cells, with a significantly higher proportion of cells at $\mathrm{G}_{0} / \mathrm{G}_{1}(\mathrm{P}<0.01)$. Consistently, in vivo data also demonstrated that tumor growth was significantly inhibited in mice injected with HT-29-shSTAT3 cells. MVD was $9.80 \pm 3.02$ in the HT-29-shSTAT3 group, significantly less than that of the control group $(P<0.01)$. mRNA and protein levels of VEGFA and MMP2 in the HT-29-shSTAT3 group were significantly lower than in the control group $(P<0.05)$, but no significant difference was observed in the mRNA or protein level of FGF2 ( $P>0.05)$. Taken together, these results demonstrate that STAT3 signaling is important to the growth of CRC and promotes angiogenesis by regulating VEGFA and MMP2 expression.
\end{abstract}

Key words: STAT3; RNA interference; Colorectal carcinoma, Angiogenesis; VEGFA; MMP2; FGF2

\section{Introduction}

Colorectal carcinoma (CRC) is the third most common malignant neoplasm worldwide and the second leading cause of cancer-related deaths (1). Despite recent advances in diagnostic and therapeutic measures, the prognosis of colorectal cancer patients remains poor. Although significant progress has been made in defining the molecular mechanisms of CRC development, the specific molecular regulation pathways involved in CRC progression are still largely unknown. In order to develop new therapeutic strategies, it is critical to understand the mechanism of progression of CRC.

It is well known that all tumors undergo neovascularization, or angiogenesis, in order to acquire nutrients for continuing growth and metastatic spread. Not surprisingly, angiogenesis is a complex multistage process and is regulated by the balance between angiogenic and angiostatic factors (2). Recently, more studies have shown that signaling transduction and activation of transcription 3 (STAT3), a key signaling transduction protein and an oncoprotein, appears to play a central role in modulating angiogenesis in various cancers including melanoma, breast cancer, gastric cancer, and hepatocellular carcinoma (3-6).

The STAT3 protein was originally discovered as a latent cytoplasmic transcription factor in response to extracellular signaling proteins, especially cytokines and growth factors. Upon activation, STAT3 protein is phosphorylated on a tyrosine residue, leading to the formation of homo- or heterodimers and translocation to the nucleus, where it

Correspondence: W.F. Qian, Department of General Surgery, Suzhou Hospital Affiliated to Nanjing Medical University, 215002 Suzhou, China. E-mail: qianweifeng163@163.com

Received April 30, 2011. Accepted October 13, 2011. Available online October 28, 2011. Published November 28, 2011. 
activates transcription of the target genes of proliferation, anti-apoptosis, or cell cycle progression by binding directly to high-affinity DNA binding sites or by associating with other transcription factors. In addition to its diverse biological functions involved in cell proliferation, differentiation, apoptosis, inflammation, and oncogenesis, accumulating evidence suggests that STAT3 also plays an important role in angiogenesis in both physiological and pathological situations (7-13). Several studies have reported that STAT3 directly regulates vascular endothelial growth factor A (VEGFA) expression to induce tumor angiogenesis in breast cancer, gastric cancer and hepatocellular carcinoma $(4,5,14)$. In pancreatic cancer, STAT3-targeting RNA interference (RNAi) inhibits angiogenesis by suppressing the expression of matrix metalloproteinase-2 (MMP2) (15). In human melanoma brain metastases, activated STAT3 contributes to tumor angiogenesis by regulating the expression of basic fibroblast growth factor (FGF2) (3). These results indicate that STAT3 signaling interferes with the process of angiogenesis in various cancers through different mechanisms.

Recent studies have shown that STAT3 was constitutively activated in CRC cell lines (16). Suppression of STAT3 expression by small interfering RNA (siRNA) inhibited cell invasion ability and induced anoikis in CRC cells (17). Clinical data also showed that in colorectal adenocarcinoma, the phospho-Tyr-STAT3 ( $p$-STAT3) level was significantly correlated with the depth of tumor invasion, venous invasion, lymph node metastasis, and increasing stages of the Dukes' classification $(18,19)$. Taken together, these findings demonstrate that the STAT3 pathway plays a critical role in the progression of CRC (20-22). However, it is unknown whether STAT3 signaling contributes to angiogenesis in CRC.

In the present study, we investigated the possible mechanism of STAT3 signaling and its effect on angiogenesis in CRC after inhibiting STAT3 expression with lentivirusmediated short hairpin RNA (shRNA).

\section{Material and Methods}

\section{Material}

The HT-29 and 293T cell lines were purchased from the Institute of Biochemistry and Cell Biology, Shanghai Institute for Biological Sciences, Chinese Academy of Science. Fifteen syngeneic female BALB/c nude mice ( 6 weeks old) were provided by the Animal Center Laboratory of Soochow University and were housed under specific-pathogen-free conditions. The use of animals in this study was approved by the Animal Ethics Committee of Soochow University and the protocol of animal treatment was approved by the Institutional Animal Care and Use Committee.

PRMI-1640 was purchased from Invitrogen (USA); fetal bovine serum was purchased from Hyclone (USA); trypsin was purchased from Sigma (USA); pRNAT-U6.2/Lenti lentivirus vector and packaging system (pMD1g-pRRE, pRsv-REV and pMD2G) were purchased from Invitrogen; Trizol reagent and lipofectamine 2000 were purchased from Invitrogen; oligo(dT), RNase inhibitor, Taq Dnase, dNTP mixture $(10 \mathrm{mM}), 10 X$ PCR buffer and $\mathrm{MgCl}_{2}(25 \mathrm{mM})$ were purchased from Promega (China); M-MLV reverse transcriptase and SYBR Green Real-Time PCR Master Mix kit were purchased from Toyobo (Japan); Nuclear-Cytosol Extraction Kit was purchased from KeyGen Biotech (China); STAT3, p-STAT3, VEGFA, MMP2, FGF2, and GAPDH antibodies were purchased from Santa Cruz (USA); CD34 antibody, goat anti-rabbit IgG-horseradish peroxidase, and antigen-removing solution of sodium citrate were purchased from Boshide (China).

\section{Construction of the recombinant lentivirus vector pRNAT-shSTAT3 and preparation of recombinant lentiviruses}

According to a previous screening study (23), we chose the site 2092-2112 of STAT3 (GeneBank accession No. NM_003150) as the target site of RNAi. The STAT3 hairpin oligos were synthesized (Invitrogen) and annealed. The sense was 5'-GATCCCGTCTTACCGCTG ATGTCCTTCTITGATATCCGAGAAGGACATCAGCGG TAAGATTTTTTCCAAC-3' and the anti-sense was 5'-GTT GGAAAAAATCTTACCGCTGATGTCCTTCTCGGATATC AAAGAAGGACATCAGCGGTAAGACGGGATC-3'). Anontargeting shRNA unrelated to human gene sequences was used as a negative control. The DNA fragments corresponding to the target gene were amplified by PCR. The up (+) position is a BamHI restriction site and the down (-) position is an Xhol restriction site. The PCR product and pRNATU6.2/Lenti lentivirus vector driven by the U6 promoter and carrying the reporter gene, enhanced green fluorescence protein (GFP), were mixed together and digested with $\mathrm{BamHI}$ and Xhol. A T4 ligase reaction system was used to connect the appropriately digested oligonucleotide and expression vector in recombinant clone ligation buffer. Competent $\mathrm{DH} 5 \alpha$ cells were transformed with the ligated products and allowed to grow in culture. The correct positive clones were confirmed by sequencing analysis (Shanghai Invitrogen, Co., China).

The recombinant lentiviruses were produced by transient transfection of 293T cells according to standard protocols (24). Briefly, subconfluent $293 \mathrm{~T}$ cells on $10-\mathrm{cm}$ plates were cotransfected with $20 \mu \mathrm{g}$ of a plasmid vector, $15 \mu \mathrm{g}$ pMD1g-pRRE, $10 \mu \mathrm{g}$ pRsv-REV, and $7.5 \mu \mathrm{g}$ pMD2G by calcium phosphate precipitation. After $16 \mathrm{~h}$ the medium was changed, and recombinant lentiviruses were harvested $24 \mathrm{~h}$ later.

\section{Transduction with recombinant lentiviruses and generation of stable cell lines}

For transduction, HT-29 cells were plated onto a $3.5-\mathrm{cm}$ dish and allowed to adhere for $16 \mathrm{~h}$. Cells were infected 
with either the recombinant STAT3-shRNA lentiviruses or the non-targeting shRNA recombinant lentiviruses in complete medium for $48 \mathrm{~h}$ (MOI 1:20). Cells were washed and transferred to complete medium for $48 \mathrm{~h}$. After selection by fluorescence-activated cell sorting using GFP as the marker, the transfected cells were obtained and expanded into stable cell lines. Cells were then harvested for the subsequent experiments. The HT-29 cells transfected with the recombinant STAT3-shRNA lentivirus were named HT-29shSTAT3 cells, while cells transfected with the recombinant non-targeting shRNA lentiviruses were named HT-29-GFP cells. The parental HT-29 cells as well as HT-29-GFP cells served as controls.

\section{Quantitative real-time PCR}

Total RNA was extracted from cells of each group (HT29, HT-29-GFP and HT-29-shSTAT3) using Trizol reagent according to manufacturer instructions. Two micrograms RNA was reverse transcribed in a $20-\mu \mathrm{L}$ reaction solution containing $10 \mathrm{U}$ M-MLV reverse transcriptase and $0.5 \mu \mathrm{g}$ oligo(dT) primer. Quantitative real-time PCR, using SYBR Green Real-Time PCR Master Mix kit, was performed according to the manufacturer protocol. The STAT3 gene was amplified using specific oligonucleotide primers and the housekeeping gene GAPDH was used as the internal standard. The sequences of the STAT3 primers were sense 5'-GAGGACTGAGCATCGAGCA-3', antisense 5'-CATGTGATCTGACACCCTGAA-3', and the size of the products was $85 \mathrm{bp}$. The sequences of the GAPDH primers were sense 5'-ACTCCTCCACCTTTGACGC-3', antisense 5'-CTCTTCCTCTTGTGCTCTTGC-3', and the size of the products was $180 \mathrm{bp}$. The data were quantified by the 2-DDCT method. All analyses were performed in triplicate.

\section{Western blot analysis}

Cell lysates were prepared with the Nuclear-Cytosol Extraction Kit, proteins were partially separated by $10 \%$ SDS-PAGE and blotted onto a membrane. After blocking at $37^{\circ} \mathrm{C}$ in $5 \%$ milk in phosphate-buffered saline (PBS) containing $0.1 \%$ Tween 20 for $2 \mathrm{~h}$, membranes were incubated with primary antibody against STAT3, p-STAT3 and GAPDH at $4^{\circ} \mathrm{C}$ overnight. After extensive washing, immunocomplexes were detected with HRP-conjugated secondary antibodies at room temperature. An enhanced chemiluminescence kit was used for detection. The amount of GAPDH detected was used as a normalization control for protein loading.

\section{Cell proliferation assay and cell cycle analysis}

Cells were plated onto 96well microwell plates at a density of 1000 cells/well. Cell prolifera- tion was determined by the MTT assay to count cells at $570 \mathrm{~nm}$ absorbance $\left(A_{570}\right)$ on a multiwell plate reader. For cell cycle analysis, cells were harvested and stained with propidium iodide to analyze DNA content by flow cytometry.

\section{In vivo experiments}

Six-week-old syngeneic female BALB/c nude mice were randomly divided into three groups: HT-29, HT-29-GFP, and HT-29-shSTAT3 (5 mice per group). Cells were suspended in PBS and $1 \times 10^{7}$ cells were injected subcutaneously into the right back of the mice. Tumor size was calculated every 5 days using the simplified formula $\left(L \times W^{2} \times 0.5\right)$. On day 30 , all mice were killed and tumor tissue was removed for further study.

\section{Histopathological and immunohistochemical examination}

Tissues were fixed in $10 \%$ neutral formalin, embedded in paraffin and cut into $5-\mu \mathrm{m}$ thick sections. Hematoxylin and eosin (HE) staining was performed for histopathological analysis. In addition, 5- $\mu$ m thick sections of formalin-fixed, paraffin-embedded specimens were stained with anti-CD34 antibodies (1:100) according to the manufacturer protocol for immunohistopathological analysis. Microvessel density (MVD) in the tumor samples was determined by the method of Weidner (25). The immunostained sections were scanned at low magnification (40X) and the tumor area with the highest density of distinctly highlighted microvessels ("hot spot") was selected. MVD was then determined in the hot spot by counting all vessels at $200 \mathrm{X}$ magnification, and the mean of the counts for three fields was calculated. Large vessels with thick muscular walls were excluded from the counts, and vessel lumens were not necessary for a structure to be defined as a vessel.

\section{Quantitative real-time PCR and Western blot analysis}

Tumor RNA of each of the 5 nude mice in each group was isolated and taken for quantitative real-time PCR of VEGFA, MMP2 and FGF2. The expression of the housekeeping gene GAPDH was used as an internal standard. The real-time PCR primer sequences are shown in Table 1. Similarly, tumor tissue protein was exacted and subjected to Western blot analysis of VEGFA, MMP2 and FGF2 according to manufacturer instructions.

Table 1. Real-time PCR primer sequences.

\begin{tabular}{lllc}
\hline Gene & Sense & Antisense & Length (bp) \\
\hline VEGFA & GAACTTTCTGCTGTCTTGGGT & TCTCGATTGGATGGCAGTA & 168 \\
MMP2 & TCCGCTGCATCCAGACTTCC & GGGGTTTGCCCGGCTCAG & 155 \\
FGF2 & CCCAGAAACCCGAGCGAGT & GGCACCGCGTCCGCTAATC & 142 \\
GAPDH & ACTCCTCCACCTTTGACGC & CTCTTCCTCTTGTGCTCTTGC & 180 \\
\hline
\end{tabular}




\section{Statistical analysis}

The statistical significance of the means was calculated by one-way ANOVA, followed by the Student-NewmanKeuls test for multiple comparisons. $\mathrm{P}<0.05$ was required for statistical significance. All computations were carried out using the SPSS13.0 statistical program.

\section{Results}

\section{Creation of stable cell lines and inhibition of the expression and activity of STAT3 by STAT3 shRNA in HT-29-shSTAT3 cells}

The recombinant lentivirus vector was successfully constructed and confirmed by DNA sequencing. After the recombinant lentivirus vector was packaged in 293T cells, recombinant lentiviruses were obtained in the supernatant at a titer of $2 \times 10^{7} \mathrm{TU} / \mathrm{mL}$. After being infected with the recombinant lentiviruses carrying the reporter gene GFP, the transfected cells expressed GFP proteins. The proportion of transfected HT-29 cells was analyzed under a fluorescence microscope. The results indicated that $85 \% \mathrm{HT}-29$ cells were transfected with the recombinant lentiviruses (Figure 1). Our data suggested that the lentivirus shRNA vector pRNATU6.2/Lenti was highly efficient in infecting HT-29 cells. After fluorescence-activated cell sorting, the HT-29-shSTAT3 line and HT-29-GFP cell lines were obtained.

STAT3 mRNA expression in each cell group was analyzed by real-time PCR. The results demonstrated that endogenous STAT3 mRNA of the HT-29-shSTAT3 cells was markedly decreased compared to HT-29-GFP and HT-29 cells (Figure 2A). Consistently, as shown in Figure 2B, the expression of STAT3 protein in HT-29-shSTAT3 cells was down-regulated compared to HT-29-GFP and HT-29 cells. To determine the levels of $\mathrm{p}$-STAT3 protein, we performed Western blot analysis using antibodies against p-STAT3. As shown in Figure 2B, p-STAT3 was also markedly de-
A

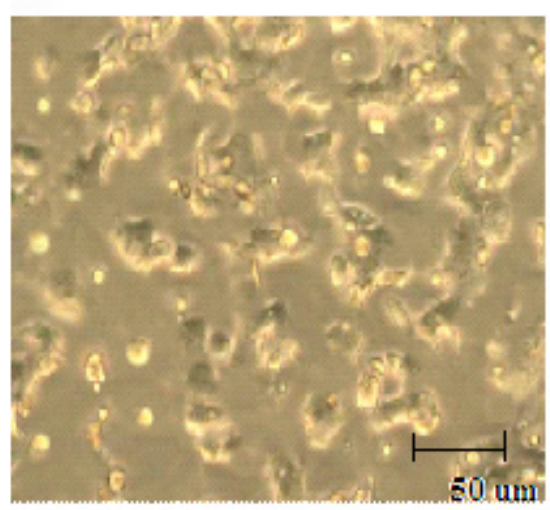

C

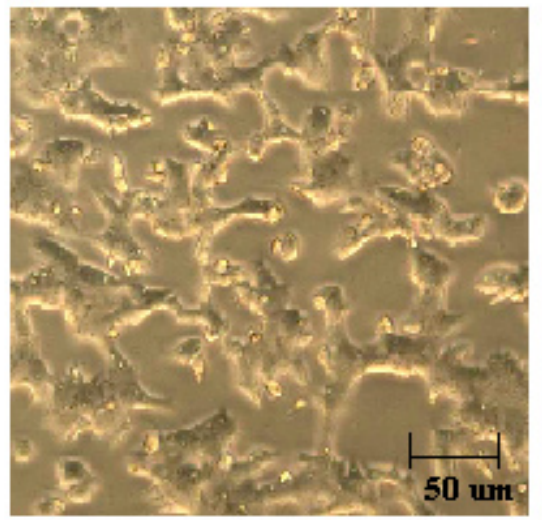

B

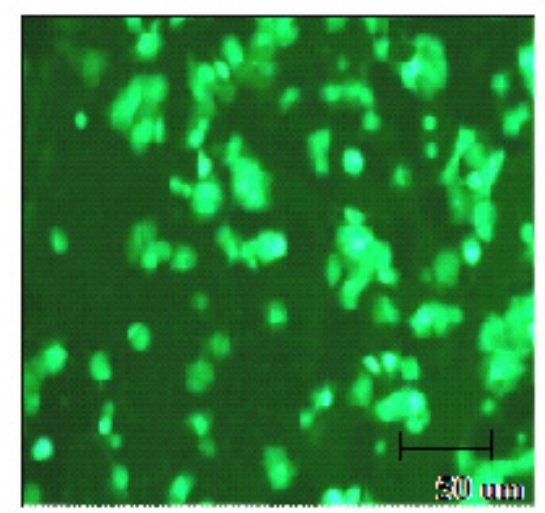

D

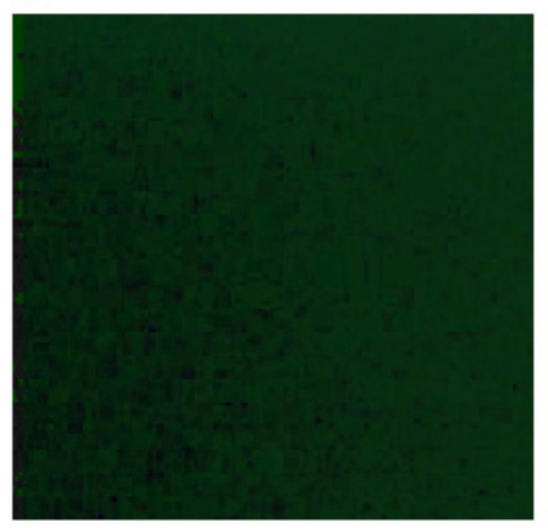

Figure 1. Micrographs of HT-29 cells infected with recombinant lentiviruses. After being infected with the recombinant lentiviruses carrying the reporter gene GFP, the transfected cells expressed GFP proteins and could be observed under a fluorescence microscope. The results indicated that $85 \% \mathrm{HT}-29$ cells were transfected with the recombinant lentiviruses. $A$ and $C$, Light microscopy; $B$ and $D$, fluorescence microscopy. Magnification bars: $50 \mu \mathrm{m}$. 
creased in HT-29-shSTAT3 cells after STAT3 silencing by RNAi. This indicated that STAT3 shRNA reduced not only the steady-state levels of total STAT3 protein, but also the levels of tyrosine-phosphorylated STAT3 protein in HT-29shSTAT3 cells.

\section{STAT3 shRNA inhibited cell growth and induced $\mathrm{G}_{0} / \mathrm{G}_{1}$ arrest}

To determine whether STAT3 down-regulated by RNAi had an inhibitory effect on HT-29 cell growth, cell proliferation rate was determined by MTT assay. As shown in Figure 3A, inhibition of STAT3 expression by RNAi suppressed the growth of HT-29-shSTAT3 cells. Cell cycle analysis (Figure $3 B$ ) showed that the proportion of $\mathrm{G}_{0} / \mathrm{G}_{1}$ of $\mathrm{HT}-29$ cells and HT-29-GFP cells was $38.65 \pm 2.33$ and $38.48 \pm 1.63 \%$, respectively. The proportion of $\mathrm{G}_{0} / \mathrm{G}_{1}$ of HT-29-shSTAT3 cells was $68.73 \pm 2.88 \%$, significantly increased compared to HT-29-GFP and HT-29 cells $(P<0.01)$.

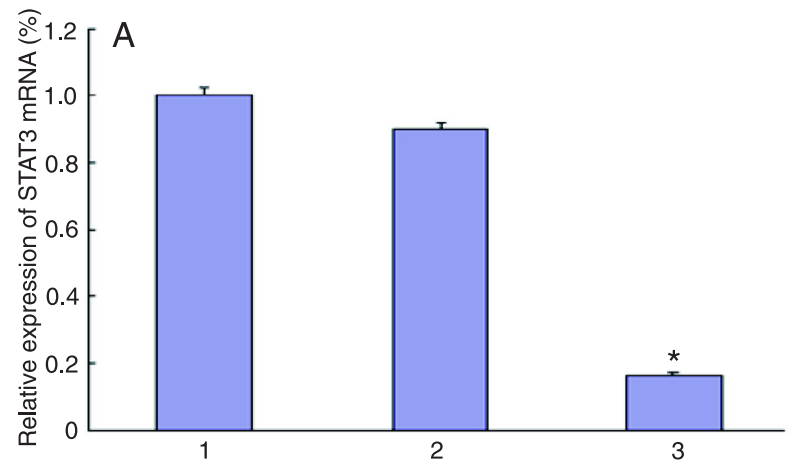

\section{Therapeutic effect on tumor-bearing nude mice}

In nude mice, HT-29 and HT-29-GFP cells rapidly formed tumors after injection. However, tumors grew slowly in the HT-29-shSTAT3 nude mouse group, with a smaller size than those in the HT-29 and HT-29-GFP groups from day 15 after inoculation $(P<0.05)$. From day 25 after injection there was a very significant difference $(P<0.01)$. Although the average tumor size in the HT-29-GFP group was smaller than that in the HT-29 group, the difference was not significant (Figure 4).

\section{Effect of STAT3 suppression on histopathology and immunohistochemistry}

$\mathrm{HE}$-stained sections were examined under a light microscope (Figure 5). In the HT-29 and HT-29-GFP groups there were many heterokaryocytes and blood vessels. The MVD of the two groups was $28.73 \pm 5.11$ and $27.60 \pm 4.27$, respectively (Figure 5). In contrast, in the HT-29-shSTAT3 group there were few blood vessels, with an MVD of 9.80

B

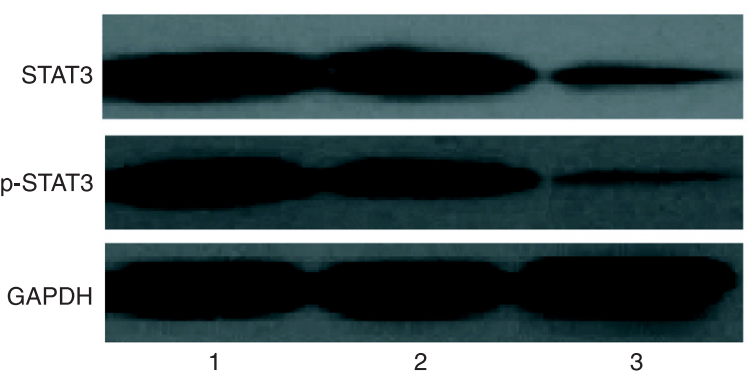

Figure 2. A, STAT3 mRNA expression in cells of each group. Group 1, HT-29 cells; group 2, HT-29-GFP cells; group 3, HT-29-shSTAT3 cells. Data are reported as means $\pm \mathrm{SEM}(\mathrm{N}=3)$. ${ }^{*} \mathrm{P}<0.01$, significantly different from the other two groups (ANOVA). $B$, Expression and activation of STAT3 proteins in cells of each group. Lane $1=$ HT-29 cells; lane $2=$ HT-29-GFP cells; lane $3=$ HT-29-shSTAT3 cells.
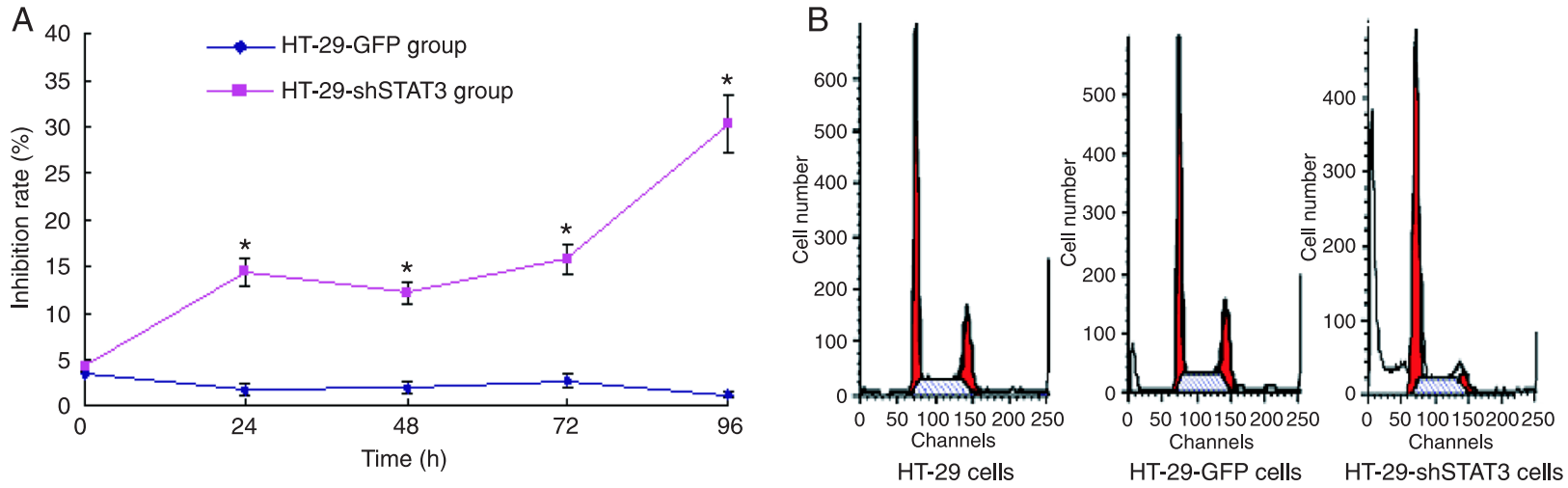

Figure 3. $A$, Growth inhibition curves of HT-29-shSTAT3 and HT-29-GFP cells. Data are reported as means $\pm \mathrm{SEM}(\mathrm{N}=3)$. ${ }^{*} \mathrm{P}<$ 0.01 , significantly different from the HT-29-GFP group (ANOVA). $B$, Cell cycle of each cell group. Cell cycle analysis showed that the proportion of $\mathrm{G}_{0} / \mathrm{G}_{1}$ of $\mathrm{HT}-29$ cells and HT-29-GFP cells was $38.65 \pm 2.33$ and $38.48 \pm 1.63 \%$, respectively. The percentage of $\mathrm{HT}-29-$ shSTAT3 cells at $\mathrm{G}_{0} / \mathrm{G}_{1}$ was $68.73 \pm 2.88 \%$, much higher than that of $\mathrm{HT}-29$-GFP cells and $\mathrm{HT}-29$ cells $(\mathrm{P}<0.01)$. 
\pm 3.02 , significantly lower than that of the HT-29 or HT-29GFP group $(P<0.01)$.

\section{Expression of VEGFA, MMP2 and FGF2 in tumors}

The mRNA expression of VEGFA, MMP2 and FGF2 in tumors was analyzed by quantitative real-time PCR (Figure $6 \mathrm{~A})$. The protein level of the target genes was determined by Western blot analysis (Figure 6B). In the HT-29-shRNA group, the mRNA and protein levels of VEGFA and MMP2 were significantly lower compared to the HT-29 and HT29-GFP groups $(P<0.05)$, but no significant change was observed in the mRNA or protein level of FGF2 among these three groups $(P>0.05)$.

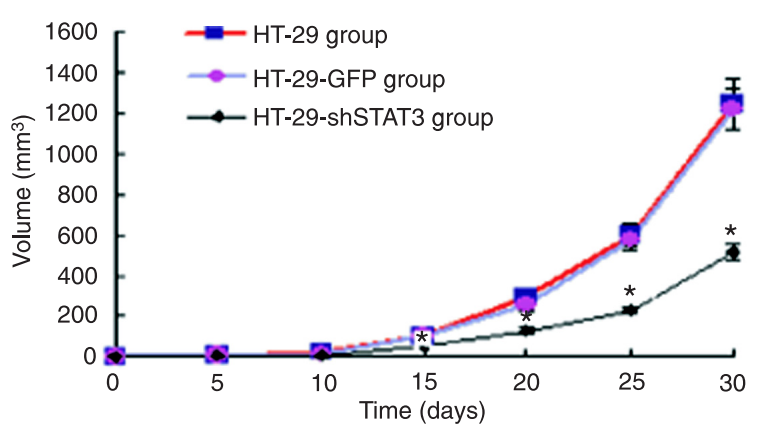

Figure 4. Change of tumor volumes in each group. ${ }^{*} \mathrm{P}<0.05$ vs HT-29 and HT-29-GFP groups (ANOVA).
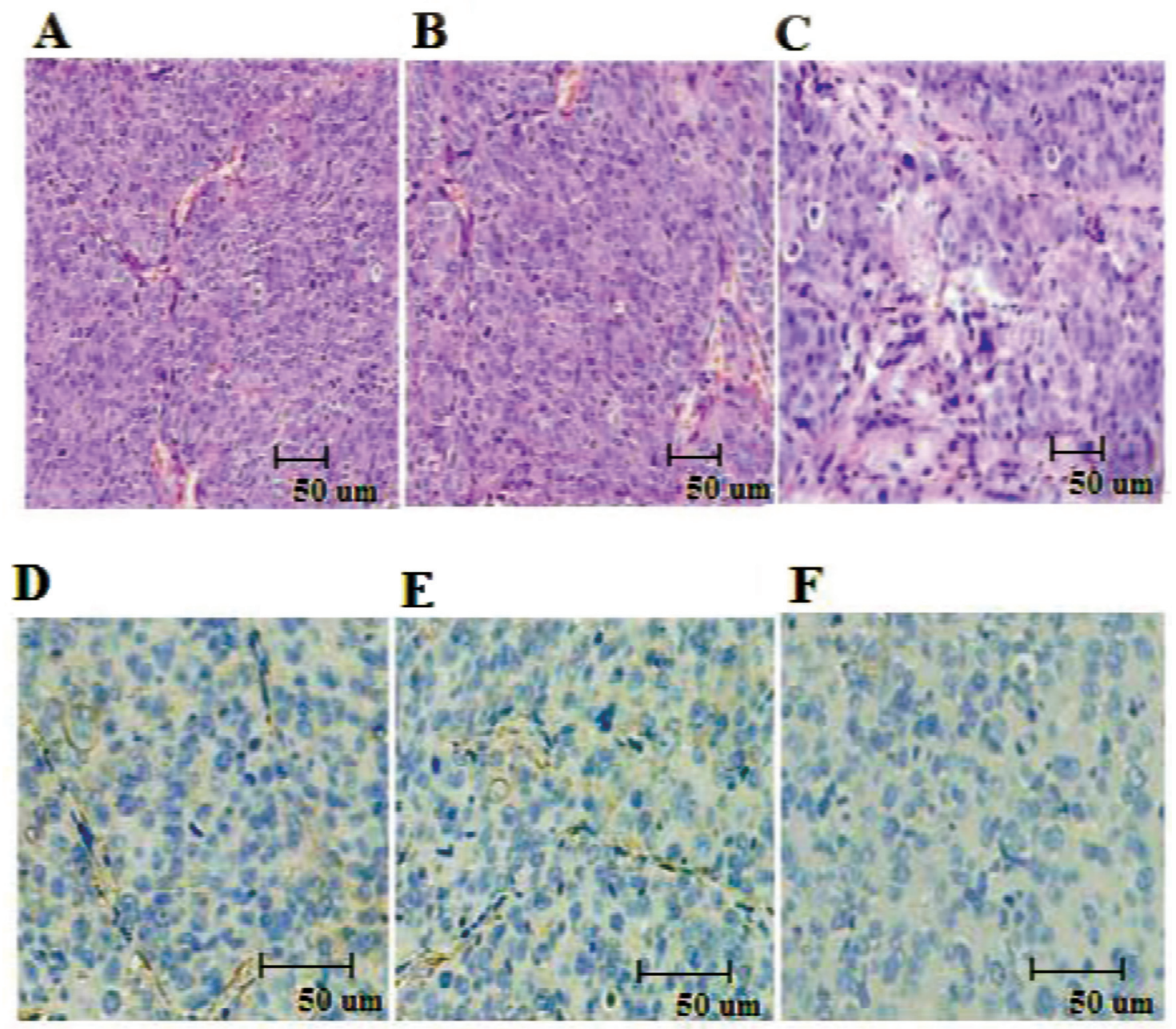

Figure 5. HE staining and CD34 immunohistochemical analysis. A, HE staining of the HT-29; B, HT-29-GFP; $C$, HT-29shSTAT3 groups. In $A$ and $B$, there were many heterokaryocytes and blood vessels. However, in $C$ there were few blood vessels. $D, E$, and $F, C D 34$ immunohistochemical analysis in the three groups, respectively. In $D$ and $E$, microvessel density was higher than in $F$. Magnification bars: $50 \mu \mathrm{m}$. 

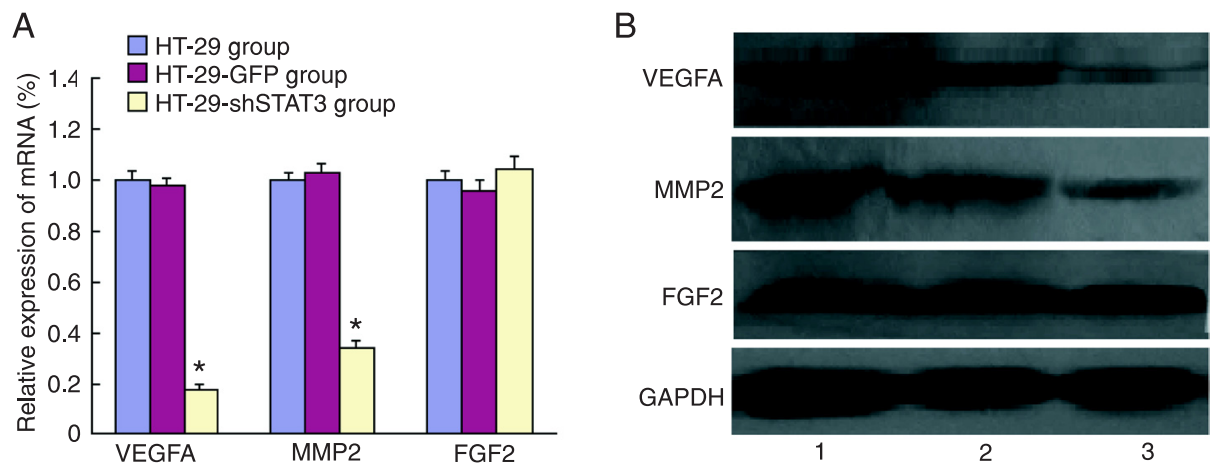

Figure 6. $A$, VEGFA, MMP2 and FGF2 mRNA expression of each group. Data are reported as means \pm SEM $(N=5)$. ${ }^{*} P<0.05$, significantly different from the other two groups (ANOVA). $B$, Expression of VEGFA, MMP2 and FGF2 proteins (an example of a mouse tumor sample from each group). Lane 1 = HT-29 group; lane 2 = HT-29-GFP group; lane 3 = HT-29-shSTAT3 group.

\section{Discussion}

STAT family proteins were originally identified as key components of cytokine signaling pathways that regulate gene expression (26). Among the 7 members of the mammalian STAT family, STAT3 is the one most strongly implicated in oncogenesis (27). Many studies have suggested that persistently activated STAT3 participates in oncogenesis through disregulation of genes encoding the antiapoptotic proteins bcl-xL, mcl-1, and bcl-2, as well as the proliferation-associated proteins cyclin D1 and c-Myc (28-30). In addition, STAT3 is able to regulate the innate and adaptive immune responses during cancer development (31). Recently, the constitutively activated STAT3 has been reported to induce tumor angiogenesis in various cancers including melanoma, breast cancer, gastric cancer, and hepatocellular carcinoma (3-6). It is not known if STAT3 signaling contributes to angiogenesis in CRC.

In order to investigate the possible mechanism of STAT3 signaling and its effect on angiogenesis in CRC after inhibiting STAT3 expression, we first constructed the HT-29-shSTAT3 cell line successfully by lentivirus-mediated RNAi. The siRNA can be used effectively in vitro and in vivo to suppress gene expression in eukaryotic cells (32), but the inhibitory effect of siRNAs on target gene expression only lasts a few days. Recently, investigators have learned that a lentivirus vector can integrate stably into the chromosomes of their target cells, a likely requisite for long-term expression, and transduce nondividing cells. Thus, lentivirus vector-mediated siRNA can inhibit target gene expression for a long time. Therefore, a lentivirus siRNA vector is an attractive tool, which can inhibit gene expression with high efficiency and has become a new tool for human gene therapy. Our data demonstrate that the rate of lentivirus vector transfected to HT-29 cells was $85 \%$. Quantitative real-time PCR analysis and Western blot assay indicated that the expression of STAT3 in HT-29 cells transfected with STAT3 shRNA was significantly suppressed at both the mRNA and protein level. The present data show that STAT3 shRNA inhibited not only STAT3 expression but also the activity of STAT3 in HT-29 cells.

Accumulating evidence has demonstrated that STAT3 plays an important role in cell proliferation of some cancers (33). The mechanism by which STAT3 participates in cell proliferation is based on induction of cell cycle genes, such as cyclin D1, c-Myc, etc. Both cyclin D1 and c-Myc respond to mitogenic signaling and are required for progression of cells from the $G_{1}$ to the $S$ phase of the cell cycle. Here, in order to determine whether STAT3 regulated HT-29 cell proliferation, the cell growth rate and cell cycle of HT-29shSTAT3 cells were analyzed. The results showed that down-regulation of STAT3 also inhibited the growth of HT29-shSTAT 3 cells and induced $G_{0} / G_{1}$ arrest of these cells. In addition, the present in vivo study showed that tumors in the HT-29-shSTAT3 group grew slowly, with a smaller size than in the HT-29 and HT-29-GFP groups. This clearly indicated that STAT3 signaling had an important effect on tumor growth in CRC-bearing nude mice.

For tumor growth, angiogenesis is essential and important, a process that involves endothelial cell proliferation, selective degradation of the basement membrane and the surrounding extracellular matrix, endothelial cell migration, and the formation of a tubular structure. Malignant tumors depend on capillary proliferation to maintain the oxygen and nutrient supply for their continuous growth. Numerous lines of evidence have shown that angiogenesis, as quantitated according to MVD, which can reflect the status of angiogenesis in tumor tissue, plays a significant clinicopathologic role in cancer patients $(34,35)$. In CRC, MVD is an important, highly significant and accurate prognostic factor $(36,37)$. The MVD method, i.e., counting microvessels in hot spots, has been the preferred method (25). In order 
to count microvessels, visualization of endothelial cells by antibodies specifically targeting them is essential. At present, CD34, CD31 and factor VIII are the antibodies most commonly used for this purpose. In the present study, the results of CD34 immunohistochemistry showed that MVD was significantly lower in the HT-29-shSTAT3 groups than in the other two groups, implying that STAT3 signaling has an important effect on angiogenesis in CRC.

Angiogenesis is a complex multistage process and is regulated by the balance between many stimulatory and inhibitory factors. In CRC, studies have shown that some proangiogenic factors, such as VEGFA, MMP2 and FGF2, play important roles in angiogenesis (37-39). In breast cancer, gastric cancer and hepatocellular carcinoma, STAT3 signaling controls VEGFA expression $(4,5,14)$. In pancreatic cancer, STAT3 signaling regulates the expression of MMP2 (15), and in human melanoma brain metastases, activated Stat3 regulates the expression of FGF2 (3). These results demonstrate that constitutive STAT3 signaling contributes to tumor angiogenesis through multiple mechanisms. In order to probe the possible mechanism of the STAT3 signaling pathway underlying angiogenesis in CRC, we determined the expressions of VEGFA, MMP2 and FGF2. The result of quantitative realtime PCR analysis and Western blot assay showed that the expression of VEGFA and MMP2 was significantly downregulated in xenografts originating from HT-29-shSTAT3

\section{References}

1. Jemal A, Murray T, Samuels A, Ghafoor A, Ward E, Thun MJ. Cancer statistics, 2003. CA Cancer J Clin 2003; 53: 5-26.

2. Bergers $G$, Benjamin LE. Tumorigenesis and the angiogenic switch. Nat Rev Cancer 2003; 3: 401-410.

3. Xie TX, Huang FJ, Aldape KD, Kang SH, Liu M, Gershenwald JE, et al. Activation of stat3 in human melanoma promotes brain metastasis. Cancer Res 2006; 66: 3188-3196.

4. Hsieh FC, Cheng G, Lin J. Evaluation of potential Stat3regulated genes in human breast cancer. Biochem Biophys Res Commun 2005; 335: 292-299.

5. Gong W, Wang L, Yao JC, Ajani JA, Wei D, Aldape KD, et al. Expression of activated signal transducer and activator of transcription 3 predicts expression of vascular endothelial growth factor in and angiogenic phenotype of human gastric cancer. Clin Cancer Res 2005; 11: 1386-1393.

6. Wang XH, Liu BR, Qu B, Xing H, Gao SL, Yin JM, et al. Silencing STAT3 may inhibit cell growth through regulating signaling pathway, telomerase, cell cycle, apoptosis and angiogenesis in hepatocellular carcinoma: potential uses for gene therapy. Neoplasma 2011; 58: 158-171.

7. Chen Z, Han ZC. STAT3: a critical transcription activator in angiogenesis. Med Res Rev 2008; 28: 185-200.

8. Valdembri D, Serini G, Vacca A, Ribatti D, Bussolino F. In vivo activation of JAK2/STAT-3 pathway during angiogenesis induced by GM-CSF. FASEB J 2002; 16: 225-227.

9. Osugi T, Oshima Y, Fujio Y, Funamoto M, Yamashita A, Ne- cells. That is, down-regulated STAT3 signaling caused a significantly decreased expression of VEGFA and MMP2 in CRC. However, it was surprising that there was no significant difference in FGF2 expression among these three groups. On the basis of these data, we speculate that STAT3 signaling activates and promotes angiogenesis by regulating VEGFA and MMP2 expression in CRC. It is highly possible that STAT3 activation is a common signal regulating VEGFA and MMP2 expression, and VEGFA and MMP2 are the downstream target molecules of STAT3 signaling in CRC. In contrast, FGF2 may not participate in the STAT3 signaling pathway in CRC.

Our findings show that STAT3 signaling plays an important role in HT-29 cell growth. STAT3 targeting shRNA can remarkably silence the expression of the gene STAT3 in HT-29 cells, resulting in inhibition of cell growth and cell cycle arrest at the $\mathrm{G}_{0} / \mathrm{G}_{1}$ phase. In nude mice, STAT3 signaling is important for the growth of $C R C$ and can promote angiogenesis by regulating VEGFA and MMP2 expression. These studies suggest that targeting STAT3 signaling can be an important approach to the control of tumor growth and angiogenesis in CRC.

\section{Acknowledgments}

Research supported by the Suzhou Social Development Program (\#SS08033). goro S, et al. Cardiac-specific activation of signal transducer and activator of transcription 3 promotes vascular formation in the heart. J Biol Chem 2002; 277: 6676-6681.

10. Niu G, Wright KL, Huang M, Song L, Haura E, Turkson J, et al. Constitutive Stat3 activity up-regulates VEGF expression and tumor angiogenesis. Oncogene 2002; 21: 2000-2008.

11. Wei D, Le X, Zheng L, Wang L, Frey JA, Gao AC, et al. Stat3 activation regulates the expression of vascular endothelial growth factor and human pancreatic cancer angiogenesis and metastasis. Oncogene 2003; 22: 319-329.

12. Haura EB, Turkson J, Jove R. Mechanisms of disease: Insights into the emerging role of signal transducers and activators of transcription in cancer. Nat Clin Pract Oncol 2005; 2: 315-324.

13. Shailubhai K, Dheer S, Picker D, Kaur G, Sausville EA, Jacob GS. Atiprimod is an inhibitor of cancer cell proliferation and angiogenesis. J Exp Ther Oncol 2004; 4: 267-279.

14. Li J, Piao YF, Jiang Z, Chen L, Sun HB. Silencing of signal transducer and activator of transcription 3 expression by RNA interference suppresses growth of human hepatocellular carcinoma in tumor-bearing nude mice. World J Gastroenterol 2009; 15: 2602-2608.

15. Qiu Z, Huang C, Sun J, Quu W, Zhang J, Li H, et al. RNA interference-mediated signal transducers and activators of transcription 3 gene silencing inhibits invasion and metastasis of human pancreatic cancer cells. Cancer Sci 2007; 98: 
1099-1106.

16. Lin Q, Lai R, Chirieac LR, Li C, Thomazy VA, Grammatikakis I, et al. Constitutive activation of JAK3/STAT3 in colon carcinoma tumors and cell lines: inhibition of JAK3/STAT3 signaling induces apoptosis and cell cycle arrest of colon carcinoma cells. Am J Pathol 2005; 167: 969-980.

17. Fan Y, Zhang YL, Wu Y, Zhang W, Wang YH, Cheng ZM, et al. Inhibition of signal transducer and activator of transcription 3 expression by RNA interference suppresses invasion through inducing anoikis in human colon cancer cells. World J Gastroenterol 2008; 14: 428-434.

18. Kusaba T, Nakayama T, Yamazumi K, Yakata Y, Yoshizaki A, Nagayasu T, et al. Expression of p-STAT3 in human colorectal adenocarcinoma and adenoma; correlation with clinicopathological factors. J Clin Pathol 2005; 58: 833-838.

19. Ma XT, Wang S, Ye YJ, Du RY, Cui ZR, Somsouk M. Constitutive activation of Stat3 signaling pathway in human colorectal carcinoma. World J Gastroenterol 2004; 10: 15691573.

20. Windham TC, Parikh NU, Siwak DR, Summy JM, McConkey DJ, Kraker AJ, et al. Src activation regulates anoikis in human colon tumor cell lines. Oncogene 2002; 21: 7797 7807.

21. Laird AD, Li G, Moss KG, Blake RA, Broome MA, Cherrington $\mathrm{JM}$, et al. Src family kinase activity is required for signal tranducer and activator of transcription 3 and focal adhesion kinase phosphorylation and vascular endothelial growth factor signaling in vivo and for anchorage-dependent and -independent growth of human tumor cells. Mol Cancer Ther 2003; 2: 461-469.

22. Orlovsky K, Theodor L, Malovani H, Chowers Y, Nir U. Gamma interferon down-regulates Fer and induces its association with inactive Stat3 in colon carcinoma cells. Oncogene 2002; 21: 4997-5001.

23. Ruan JL, Gao Y, Guan WX, Qiao ZM, Qian WF. Construction of STAT3 lentiviral vector. J Nantong Univ Med Sci 2010; 30: 83-85.

24. Zufferey R, Nagy D, Mandel RJ, Naldini L, Trono D. Multiply attenuated lentiviral vector achieves efficient gene delivery in vivo. Nat Biotechnol 1997; 15: 871-875.

25. Weidner N. Intratumor microvessel density as a prognostic factor in cancer. Am J Pathol 1995; 147: 9-19.

26. Murray PJ. The JAK-STAT signaling pathway: input and output integration. J Immunol 2007; 178: 2623-2629.

27. Haura EB. SRC and STAT pathways. J Thorac Oncol 2006; 1: 403-405.

28. Mora LB, Buettner R, Seigne J, Diaz J, Ahmad N, Garcia $\mathrm{R}$, et al. Constitutive activation of Stat3 in human prostate tumors and cell lines: direct inhibition of Stat3 signaling induces apoptosis of prostate cancer cells. Cancer Res 2002; 62: 6659-6666.

29. Bowman T, Broome MA, Sinibaldi D, Wharton W, Pledger WJ, Sedivy JM, et al. Stat3-mediated Myc expression is required for Src transformation and PDGF-induced mitogenesis. Proc Natl Acad Sci U S A 2001; 98: 7319-7324.

30. Masuda M, Suzui M, Yasumatu R, Nakashima T, Kuratomi Y, Azuma K, et al. Constitutive activation of signal transducers and activators of transcription 3 correlates with cyclin D1 overexpression and may provide a novel prognostic marker in head and neck squamous cell carcinoma. Cancer Res 2002; 62: 3351-3355.

31. Wang T, Niu G, Kortylewski M, Burdelya L, Shain K, Zhang $S$, et al. Regulation of the innate and adaptive immune responses by Stat-3 signaling in tumor cells. Nat Med 2004; 10: 48-54.

32. Lewis DL, Hagstrom JE, Loomis AG, Wolff JA, Herweijer H. Efficient delivery of siRNA for inhibition of gene expression in postnatal mice. Nat Genet 2002; 32: 107-108.

33. Aggarwal BB, Sethi G, Ahn KS, Sandur SK, Pandey MK, Kunnumakkara $A B$, et al. Targeting signal-transducer-andactivator-of-transcription-3 for prevention and therapy of cancer: modern target but ancient solution. Ann N Y Acad Sci 2006; 1091: 151-169.

34. Weidner N, Semple JP, Welch WR, Folkman J. Tumor angiogenesis and metastasis - correlation in invasive breast carcinoma. N Engl J Med 1991; 324: 1-8.

35. Weidner N, Folkman J, Pozza F, Bevilacqua P, Allred EN, Moore DH, et al. Tumor angiogenesis: a new significant and independent prognostic indicator in early-stage breast carcinoma. J Natl Cancer Inst 1992; 84: 1875-1887.

36. Gao J, Knutsen A, Arbman G, Carstensen J, Franlund B, Sun $X F$. Clinical and biological significance of angiogenesis and lymphangiogenesis in colorectal cancer. Dig Liver Dis 2009; 41: 116-122.

37. Des Guetz G, Uzzan B, Nicolas P, Cucherat M, Morere $\mathrm{JF}$, Benamouzig R, et al. Microvessel density and VEGF expression are prognostic factors in colorectal cancer. Meta-analysis of the literature. Br J Cancer 2006; 94: 18231832.

38. Kim TS, Kim YB. Correlation between expression of matrix metalloproteinase-2 (MMP-2), and matrix metalloproteinase-9 (MMP-9) and angiogenesis in colorectal adenocarcinoma. J Korean Med Sci 1999; 14: 263-270.

39. Kos M, Dabrowski A. Tumour's angiogenesis - the function of VEGF and bFGF in colorectal cancer. Ann Univ Mariae Curie Sklodowska Med 2002; 57: 556-561. 